\title{
UNIT GROUPS OF INTEGRAL GROUP RINGS
}

\author{
VIKAS BIST \\ (Communicated by Maurice Auslander)
}

\begin{abstract}
Let $U(\mathbb{Z} G)$ be the unit group of the integral group ring $\mathbb{Z} G$. A group $G$ satisfies $(*)$ if either the set $T(G)$ of torsion elements of $G$ is a central subgroup of $G$ or, otherwise, if $x \in G$ does not centralize $T(G)$, then for every $t \in T(G), x^{-1} t x=t^{-1}$. This property appears quite frequently while studying $U(\mathbb{Z} G)$. In this paper we investigate why one encounters this property and we have also given a "unified proof" for some known results regarding this property. Further, some additional results have been obtained.
\end{abstract}

\section{INTRODUCTION}

Let $\mathbb{Z} G$ be an integral group ring of a group $G$ and let $U(\mathbb{Z} G)$ be its group of units. Denote by $T(G)$ the set of all torsion elements of $G$. A group $G$ is Hamiltonian if $G$ is nonabelian and every subgroup of $G$ is normal.

We say that a group $G$ satisfies the property $(*)$ if one of the following holds:

(a) $T(G)$ is a central subgroup of $G$.

(b) $T(G)$ is abelian, noncentral, and if $x \in G$ does not centralize $T(G)$, then $x^{-1} t x=t^{-1}$ for every $t \in T(G)$.

(c) $T(G)$ is a Hamiltonian 2-group and every subgroup of $T(G)$ is normal in $G$.

The property $(*)$ appears quite frequently while studying $U(\mathbb{Z} G)$. We list the following results:

(1) $U(\mathbb{Z} G)$ is nilpotent if and only if $G$ is nilpotent and satisfies $(*)[7$, VI.3.23].

(2) $U(\mathbb{Z} G)$ is FC if and only if $G$ is FC and $G$ satisfies (*) [7, VI.5.4].

(3) The commutator subgroup $U(\mathbb{Z} G)^{\prime}$ of $U(\mathbb{Z} G)$ is torsion if and only if $G^{\prime}$ is torsion and $G$ satisfies $(*)[1,2]$.

(4) $U(\mathbb{Z} G) / \zeta(U(\mathbb{Z} G))$ is locally finite if and only if $G / \zeta(G)$ is locally finite and $G$ satisfies $(*)$. Here $\zeta(G)$ denotes the center of $G$ [1].

(5) Let $G$ be such that $G / T(G)$ is right ordered. Then $U(\mathbb{Z} G)$ is Engel ( $n$-Engel) if and only if $G$ is Engel ( $n$-Engel) and satisfies $(*)$ [2].

The similarity in the above results motivates us to look for a "unified proof" for these results to see why $G$ always has the property $(*)$. In this process we

Received by the editors August 26, 1991 and, in revised form, April 8, 1992.

1991 Mathematics Subject Classification. Primary 16S34; Secondary 16U60. 
obtain easier proofs of the above mentioned results (particularly of (2) and (5)) and we also get some new results of a similar nature.

\section{CONDition $(*)$ IS NECESSARY}

The necessity of $(*)$ in the above results will be shown by proving that the hypothesis in each of them implies the following statements:

(I) $T(G)$ is either abelian or a Hamiltonian group and every subgroup of $T(G)$ is normal in $G$.

(II) If $x \in G$ and $t \in T(G)$ are such that $x^{-1} t x \neq t$, then $x^{-1} t x=t^{-1}$.

It is easy to see that (I) and (II) imply that if $T(G)$ is nonabelian, then it is a Hamiltonian 2-group, thus establishing $(*)$ in all cases.

If $T(U(\mathbb{Z} G))$ is a subgroup, then $T(G)$ is either an abelian or a Hamiltonian 2-group with every subgroup of $T(G)$ normal in $G$ [5]. Thus (I) holds if $U(\mathbb{Z} G)$ is one of the types as described in (1)-(4). For (5) we let $x \in G$ and $t \in T(G)$. Then $1+\alpha \in U(\mathbb{Z} G)$, where $\alpha=(1-t) x\left(1+t+\cdots+t^{O(t)-1}\right)$. Now as $U(\mathbb{Z} G)$ is Engel, for some $k$,

$$
\left(1+\alpha,{ }_{k} t^{-1}\right)=(1+\alpha, \underbrace{t^{-1}, \ldots, t^{-1}}_{k})=1,
$$

i.e.,

$$
(1-t)^{k+1} x\left(1+t+\cdots+t^{O(t)-1}\right)=0
$$

thus

$$
(1-t) x\left(1+t+\cdots+t^{O(t)-1}\right)=0 .
$$

Since $(1-t)^{2} \beta=0$ for any $\beta \in \mathbb{Z} G$ and $t \in T(G)$, it follows that $(1-t) \beta=0$ (see [7, p. 182]).

This shows that $\langle t\rangle$ is normal in $G$ and thus $T(G)$ is either abelian or a Hamiltonian group.

For (II) first note that if $G$ is nonabelian torsion, then by [4] $G$ will be a Hamiltonian 2-group, otherwise $U(\mathbb{Z} G)$ will have a noncyclic free subgroup.

We assume that $G$ is nontorsion and $T(G)$ is either abelian or a Hamiltonian group and every subgroup of $T(G)$ is normal in $G$.

Let $t \in T(G)$ be an element of order $l, x \in G$, and $x^{-1} t x \neq t$. We can assume that $x$ is of infinite order.

Now consider the rational group algebra $\mathbb{Q}\langle t\rangle$. By [7, II.2.6],

$$
\mathbb{Q}\langle t\rangle=\bigoplus \sum_{d \mid l} \mathbb{Q}\left(\xi_{d}\right) ; \quad t \in \sum_{d \mid l} \xi_{d}
$$

where $\xi_{d}$ is a primitive $d$ th root of unity. Also as $x^{-1} t x=t^{r} ;(l, r)=1$, and $t^{x}=\sum_{d \mid l} \xi_{d}^{r}$. By [7, VI.1.16], if $e$ is an idempotent $\mathbb{Q}\langle t\rangle$, then $x^{-1} e x=e$, thus $x^{-1} \mathbb{Q}\left(\xi_{d}\right) x=\mathbb{Q}\left(\xi_{d}\right)$ and $\theta_{x}: \xi_{l} \mapsto \xi_{l}^{r}$ is a $\mathbb{Q}$-automorphism of $\mathbb{Q}\left(\xi_{l}\right)$.

Let $R=\bigoplus \sum_{d \mid l} \mathbb{Z}\left[\xi_{d}\right]$. Then by [7, II.2.9], $|U(R): U(Z\langle t\rangle)|<\infty$. Now $U\left(\mathbb{Z}\left[\xi_{l}\right]\right)=\left\langle \pm \xi_{l}\right\rangle \times A$, where $A$ is free abelian of finite rank. Thus for some positive integer $s, V=U\left(\mathbb{Z}\left[\xi_{l}\right]\right)^{2 l s} \subseteq U(\mathbb{Z}\langle t\rangle)$.

Clearly $\mathscr{G}=\langle V, x\rangle$ is finitely generated and torsion free. We claim that $\mathscr{G}$ is actually abelian. 
In fact, if $\mathscr{G}$ is nilpotent, then $\mathscr{G} / \zeta(\mathscr{G})$ is torsion free as $\mathscr{G}$ is torsion free. Further, $\langle t\rangle$ is normal in $G$ so $x^{n}$ commutes with $t$ for some $n$, i.e., $x^{n} \in \zeta(\mathscr{G})$. Hence $\mathscr{G}$ is abelian. This also covers the case if $\mathscr{G}$ is Engel as a finitely generated soluble Engel group is nilpotent.

In the other cases $\left((2),(3)\right.$, and (4)) $\mathscr{G}^{\prime}$ is torsion, but since $\mathscr{G}^{\prime} \subseteq V$ and $V$ is torsion free, it follows that $\mathscr{G}$ is abelian.

Finally we have that $x$ commutes elementwise with $V$. Hence $\mathbb{Q}(V) \subseteq$ $\overline{\mathbb{Q}\left(\xi_{l}\right)}$, where $\overline{\mathbb{Q}\left(\xi_{l}\right)}$ is the fixed field of the automorphism $\theta_{x}$. Since $\operatorname{rank} U\left(\mathbb{Z}\left[\xi_{l}\right]\right)=\operatorname{rank}(V)$, by [7, II.2.10], $x^{-1} \xi_{l} x=\xi_{l}^{-1}$ and thus $x^{-1} t x=t^{-1}$.

\section{Condition $(*)$ IS SUFFICIENT}

We first prove the following lemma.

Lemma. Let $G$ be a group satisfying (*) and let $G / T(G)$ be right ordered. Then $U(\mathbb{Z} G)=\mathscr{H} G$ for some $\mathscr{H} \subseteq \zeta(U(\mathbb{Z} G))$.

Proof. By [4], $U(\mathbb{Z} G)=U(\mathbb{Z} T(G)) G$. If $T(G)$ is central, then let $\mathscr{H}=$ $U(\mathbb{Z} T(G))$. If $T(G)$ is a Hamiltonian 2-group, then $U(\mathbb{Z} T(G))= \pm T(G)[7$, II.2.1 and II.2.2] and so $U(\mathbb{Z} G)= \pm G$. Here $\mathscr{H}=\{+1,-1\}$.

Further, for any $\alpha=\sum_{t \in T(G)} \alpha(t) t$ let $\alpha^{*}=\sum_{t \in T(G)} \alpha(t) t^{-1}$. If $T(G)$ is noncentral and abelian, then for any $\alpha \in U(\mathbb{Z} T(G)), x^{-1} \alpha x=\alpha$ or $\alpha^{*}$. Therefore $\mathscr{H}=\left\{\alpha \in U(\mathbb{Z} T(G)) \mid \alpha=\alpha^{*}\right\}$ is a central subgroup of $U(\mathbb{Z} G)$.

To prove that $U(\mathbb{Z} G)=\mathscr{H} G$, we let $\alpha=\sum_{i=1}^{m} z_{i} x_{i} \in U(\mathbb{Z} T(G))$, with $\varepsilon(\alpha)=1$, where $\varepsilon$ is the augmentation map. Then

$$
\begin{aligned}
\alpha & =1+\sum_{i=1}^{m} z_{i}\left(x_{i}-1\right) \\
& =\left(\prod_{i=1}^{m} x_{i}^{z_{i}}\right)+\theta, \quad \theta \in I_{\mathbb{Z}}^{2}(T(G)) \\
& =t+\theta, \quad t=\prod_{i=1}^{m} x_{i}^{z_{i}} \in T(G) .
\end{aligned}
$$

Here $I_{\mathbb{Z}}(T(G))$ denotes the augmentation ideal of $\mathbb{Z} T(G)$. Thus $\alpha=(1+\delta) t$; $\delta=\theta t^{-1} \in I_{\mathbb{Z}}^{2}(T(G))$.

We now show that $(1+\delta)^{*}=1+\delta$, hence $1+\delta \in \mathscr{H}$. Let $\beta=1+\delta$ and $\gamma=\beta^{*} \beta^{-1}$. Then $\gamma^{*} \gamma=1$ and $\varepsilon(\gamma)=1$ imply that $\gamma=x \in T(G)$. This gives the result that $x=\beta^{*} \beta^{-1} \in\left(1+I_{\mathbb{Z}}^{2}(T(G))\right) \cap T(G)=T(G)^{\prime}=1$. Hence $\beta^{*}=\beta$ implies that $U(\mathbb{Z} T(G))=\mathscr{H} T(G)$ and $U(\mathbb{Z} G)=\mathscr{H} G$.

Now due to the previous lemma, the converse follows easily since $U(\mathbb{Z} G)=$ $\mathscr{H} G$. For $\alpha_{1}, \ldots, \alpha_{n} \in U(\mathbb{Z} G)$, there are $\beta_{1}, \ldots, \beta_{n}$ in $\mathscr{H}$ and $x_{1}, \ldots, x_{n}$ in $G$ such that $\alpha_{k}=\beta_{k} x_{k}, k=1, \ldots, n$. Thus $\left(\alpha_{1}, \ldots, \alpha_{n}\right)=\left(x_{1}, \ldots, x_{n}\right)$. Also $\mathscr{H} \zeta(G) \subseteq \zeta(U(\mathbb{Z} G))$, thus $U(\mathbb{Z} G) / \mathscr{H} \zeta(G) \cong G / \zeta(G)$.

\section{Further RESUlts}

The discussions of $\S \S 2$ and 3 show that if $P$ is a group-theoretical property which is subgroup closed, then $U(\mathbb{Z} G) \in P$ implies that $G \in P$ and satisfies (*) if it can be proved that $T(G)$ is either abelian or a Hamiltonian group and 
every subgroup of $T(G)$ is normal in $G$. Then the group $\mathscr{G}$ defined in $\S 2$ is abelian. To show the converse, the lemma of $\S 3$ should hold. Thus we have the following straightforward results, which we state as corollaries.

Corollary 1. $U(\mathbb{Z} G)$ is locally nilpotent if and only if $G$ is locally nilpotent and satisfies $(*)$.

Corollary 2. $U(\mathbb{Z} G)$ is locally $F C$ if and only if $G$ is locally $F C$ and satisfies (*).

Corollary 3. $U(\mathbb{Z} G)$ is hypercentral if and only if $G$ is hypercentral and satisfies $(*)$.

This is so because hypercentral groups are locally nilpotent.

Corollary 4. $U(\mathbb{Z} G)$ satisfies the normalizer condition if and only if $G$ satisfies the normalizer condition and $(*)$.

Corollary 5. Every subgroup of $U(\mathbb{Z} G)$ is subnormal if and only if every subgroup of $G$ is subnormal and $G$ satisfies $(*)$.

Corollary 6. If $U(\mathbb{Z} G) / \zeta(U(\mathbb{Z} G))$ is torsion, then $G / \zeta(G)$ is torsion and satisfies $(*)$. Conversely, if $G / \zeta(G)$ is torsion and satisfies $(*)$ and $G / T(G)$ is right ordered, then $U(\mathbb{Z} G) / \zeta(U(\mathbb{Z} G))$ is torsion.

The following is an analogue of the theorem in [3] for integral group rings.

Theorem. $U(\mathbb{Z} G)$ is soluble and $n$-Engel if and only if $U(\mathbb{Z} G)$ is nilpotent.

Proof. Suppose that $U(\mathbb{Z} G)$ is soluble and $n$-Engel. Then $G$ is soluble and $n$-Engel and satisfies $(*)$.

It is sufficient to show that $G$ is nilpotent. Since $G / T(G)$ is torsion free soluble $n$-Engel, by [6, Corollary 7.36] $G / T(G)$ is nilpotent. Hence $\gamma_{l}(G) \subseteq$ $T(G)$ for some $l$. Here $\gamma_{l}(G)$ is the $l$ th term of the lower central chain of $G$.

If $T(G)$ is central, then $G$ is nilpotent. If $T(G)$ is noncentral, then for every $t \in T(G)$ and $x \in G,\left(t,{ }_{n} x\right)=1$. Now as $x^{-1} t x=t$ or $t^{-1}$, we have $o(t) \leq 2^{n}$. Thus $T(G)$ is of exponent at most $2^{n}$.

Again, since $x^{-1} t x=t$ or $t^{-1}$ for $t \in T(G), x \in G$, it follows that $\left(t, x_{1}, \ldots, x_{n}\right)=1$ for every $t \in T(G)$ and $x_{i} \in G, i=1, \ldots, n$. Thus $\gamma_{l+n}(G)=1$, i.e., $G$ is nilpotent.

\section{ACKNOWLEDGMENT}

The author wishes to thank the referee for his helpful suggestions and corrections which improved this paper.

\section{REFERENCES}

1. Vikas Bist, The structure of unit groups of group rings, Ph.D. thesis, 1990.

2. A. A. Bovdi, Construction of an integral group ring with trivial elements of finite order, Sibirsk. Mat. Zh. 21 (1987), 28-37.

3. J. L. Fischer, M. M. Parmenter, and S. K. Sehgal, Group rings with soluble n-Engel unit groups, Proc. Amer. Math. Soc. 59 (1976), 195-200.

4. B. Hartley and P. F. Pickel, Free subgroups in the unit groups of integral group rings, Canad. J. Math. 32 (1981), 1342-1352. 
5. C. Polcino Milies, Group rings whose torsion units form a subgroup, Proc Amer. Math. Soc. 81 (1981), 172-174.

6. D. J. S. Robinson, Finiteness conditions and generalized soluble groups, Part 2, SpringerVerlag, Berlin, 1972.

7. S. K. Sehgal, Topics in group rings, Marcel Dekker, New York, 1978.

Department of Mathematics, Panjab University, Chandigarh-160014, India 